\title{
A Combinatorial Proof of the Sum of $q$-Cubes
}

\author{
Kristina C. Garrett \\ Department of Mathematics and Computer Science \\ Carleton College, Minnesota, USA \\ kgarrett@carleton.edu \\ Kristen Hummel \\ Department of Mathematics and Computer Science \\ Carleton College, Minnesota, USA \\ hummelk@carleton.edu
}

Submitted: Nov 2, 2003; Accepted: Dec 15, 2003; Published: Jan 23, 2004

MR Subject Classifications: 05A17, 05A19

\begin{abstract}
We give a combinatorial proof of a $q$-analogue of the classical formula for the sum of cubes.
\end{abstract}

\section{Introduction}

The classic formula for the sum of the first $n$ cubes,

$$
\sum_{k=1}^{n} k^{3}=\left(\begin{array}{c}
n+1 \\
2
\end{array}\right)^{2}
$$

is easily proved by mathematical induction. Many other proofs exist that connect this simple identity to various branches of mathematics. (See [4].) The nature of the right hand side of the identity seems to suggest that a simple combinatorial proof should exist. Indeed, Benjamin and Orrison give such a proof in [2] and other combinatorial proofs are given in [3]. In this paper we will give a $q$-analogue of (1) and a bijective proof using integer partitions. We begin by reviewing a few of the basics of partition theory.

Definition 1.1. An integer partition, $\lambda$, of a positive integer $n$ is a sequence of nonincreasing positive integers $\lambda=\left(\lambda_{1}, \lambda_{2}, \ldots, \lambda_{k}\right)$ such that $\lambda_{1}+\lambda_{2}+\cdots+\lambda_{k}=n$. The $\lambda_{i}$ are the parts of the partition. The number $n$ partitioned by $\lambda$ is called the size of the partition and is denoted $\|\lambda\|$.

Another method for representing a partition $\lambda$ is the graphical representation commonly referred to as the Ferrers shape which was introduced by Sylvester who was writing 
about a proof described to him by N.M. Ferrers. The Ferrers shape of a partition is an array of boxes, left justified, in which the number of boxes in the first row is equal to the size of the first part, the number of boxes in the second row is equal to the size of the second part, etc. For example, the Ferrers diagram for the partition $\lambda=(4,4,3,2,1)$ is shown in Figure 1.

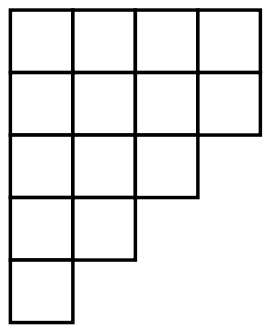

Figure 1: Ferrers Diagram for $\lambda=(4,4,3,2,1)$.

Our final bit of notation is the $q$-binomial coefficient

$$
\left[\begin{array}{l}
n \\
k
\end{array}\right]_{q}=\frac{\prod_{i=n-k+1}^{n}\left(1-q^{i}\right)}{\prod_{i=1}^{k}\left(1-q^{i}\right)}
$$

It is well known that $\left[\begin{array}{l}n \\ k\end{array}\right]_{q}$ is the generating function for partitions whose Ferrers shape fits inside a $k \times(n-k)$ box. For a more comprehesive introduction to the basics of partition theory, see [1].

\section{$2 q$-analogue of the Sum of Cubes}

In this section we introduce the following polynomial generalization of (1) along with a bijective proof.

$$
\sum_{k=1}^{n} q^{k-1}\left(\frac{1-q^{k}}{1-q}\right)^{2}\left[\frac{1-q^{k-1}}{1-q^{2}}+\frac{1-q^{k+1}}{1-q^{2}}\right]=\left[\begin{array}{c}
n+1 \\
2
\end{array}\right]_{q}^{2}
$$

The above equation is considered a " $q$-analogue" because equation (1) can be found by taking $\lim _{q \rightarrow 1^{-}}$of equation (2).

We will define two sets, $S$ and $T$, of partitions and give a weight-preserving bijection between them.

Let $S$ be the set of pairs of partitions $(\lambda, \mu)$ each of whose Ferrers shapes fit inside a $2 \times(n-1)$ box. For purposes of the bijection, we allow parts of size 0 in $\lambda$ and $\mu$. For 
example, if $\lambda=4$ then we say $\lambda_{1}=4$ and $\lambda_{2}=0$. It follows from standard results that $g(q)=\sum_{(\lambda, \mu) \in S} q^{\|\lambda\|+\|\mu\|}$ is equal to the right hand side of (2).

In order to define the set $T$, we will consider disjoint subsets, $T_{k}$. For odd $k$, let $T_{k}$ be the set of 3 -tuples $(\nu, a, b) \cup\left(\nu, a, b^{\prime}\right)$ where:

$$
\begin{aligned}
\nu & =\text { partition with at most two parts, largest part equal to }(k-1), \\
a & =j, \quad 0 \leq j \leq(k-1), \\
b & =2 l \text { with } 0 \leq l \leq \frac{(k-3)}{2} \\
b^{\prime} & =2 m \text { with } 0 \leq m \leq \frac{(k-1)}{2} .
\end{aligned}
$$

For even $k$, let $T_{k}$ be the set of 3-tuples $(\nu, a, b) \cup\left(\nu, a, b^{\prime}\right)$ where:

$$
\begin{aligned}
\nu & =\text { partition with at most two parts, largest part equal to }(k-1), \\
a & =2 j, \quad 0 \leq j \leq \frac{(k-2)}{2} \\
b & =l, \quad 0 \leq l \leq(k-2) \\
b^{\prime} & =m, \quad 0 \leq m \leq k .
\end{aligned}
$$

Let $T=\cup_{k=1}^{n} T_{k}$. Let $f(q)=\sum_{t \in T} q^{|t|}$, where $|t|=\|\nu\|+a+b$ (or $|t|=\|\nu\|+a+b^{\prime}$ respectively), be the generating function for the 3 -tuples in $T$. It is easy to verify that $f(q)$ is the left hand side of $(2)$.

We now give a weight-preserving bijection $\phi: S \rightarrow T$ in order to prove $g(q)=f(q)$ and therefore establish equation (2).

The bijection $\phi$ is defined in cases. Let $(\lambda, \mu) \in S$. We first determine $k$. Let $k=\max \left(\lambda_{1}, \mu_{1}\right)+1$. We then consider two cases depending on the parity of $k$.

1. If $k$ is odd, compare $\lambda_{1}$ and $\mu_{1}$.

(a) If $\lambda_{1} \geq \mu_{1}$, there are three subcases:

i. If $\mu_{1}$ is even, then $\phi(\lambda, \mu)=\left(\lambda, \mu_{2}, \mu_{1}^{\prime}\right)$.

ii. If $\mu_{1}$ is odd and $\mu_{2}$ is even, then $\phi(\lambda, \mu)=\left(\lambda, \mu_{1}, \mu_{2}\right)$.

iii. If $\mu_{1}$ is odd and $\mu_{2}$ is odd, then $\phi(\lambda, \mu)=\left(\lambda, \mu_{1}+1, \mu_{2}-1\right)$.

(b) If $\lambda_{1}<\mu_{1}$, there are three subcases:

i. If $\lambda_{1}$ is even, then $\phi(\lambda, \mu)=\left(\mu, \lambda_{2}, \lambda_{1}\right)$.

ii. If $\lambda_{1}$ is odd and $\lambda_{2}$ is even, then $\phi(\lambda, \mu)=\left(\mu, \lambda_{1}, \lambda_{2}^{\prime}\right)$.

iii. If $\lambda_{1}$ is odd and $\lambda_{2}$ is odd, then $\phi(\lambda, \mu)=\left(\mu, \lambda_{1}+1,\left(\lambda_{2}-1\right)^{\prime}\right)$.

2. If $k$ is even, compare $\lambda_{1}$ and $\mu_{1}$. 
(a) If $\lambda_{1} \geq \mu_{1}$, there are three subcases:

i. If $\mu_{1}$ is even, then $\phi(\lambda, \mu)=\left(\lambda, \mu_{1}, \mu_{2}\right)$.

ii. If $\mu_{1}$ is odd and $\mu_{2}$ is even, then $\phi(\lambda, \mu)=\left(\lambda, \mu_{2}, \mu_{1}^{\prime}\right)$.

iii. If $\mu_{1}$ is odd and $\mu_{2}$ is odd, then $\phi(\lambda, \mu)=\left(\lambda, \mu_{2}-1,\left(\mu_{1}+1\right)^{\prime}\right)$.

(b) If $\lambda_{1}<\mu_{1}$, there are three subcases:

i. If $\lambda_{1}$ is even, then $\phi(\lambda, \mu)=\left(\mu, \lambda_{1}, \lambda_{2}^{\prime}\right)$.

ii. If $\lambda_{1}$ is odd and $\lambda_{2}$ is even, then $\phi(\lambda, \mu)=\left(\mu, \lambda_{2}, \lambda_{1}\right)$.

iii. If $\lambda_{1}$ is odd and $\lambda_{2}$ is odd, then $\phi(\lambda, \mu)=\left(\mu, \lambda_{2}-1, \lambda_{1}+1\right)$.

We consider an example of the map $\phi$ below.

Example 2.1. Let $n=6$ and $(\lambda, \mu)=((5,3),(3,1)) \in S$. Then $k=\max \left(\lambda_{1}, \mu_{1}\right)+1=$ $5+1=6$. Since $k=6$ is even and $\lambda_{1} \geq \mu_{1}$, we are in the case 2 (a). Since both $\mu_{1}$ and $\mu_{2}$ are odd we are in the third subcase. Thus, $\phi((5,3),(3,1))=\left((5,3), 0,4^{\prime}\right) \in T$.

It is clear that $\phi$ is weight-preserving by the definition. It is also easy to check that $\phi$ is a bijection. We need to show $\phi$ is $1-1$ and onto. We will outline the proof for $k$ odd and leave the details for $k$ even to the reader. First, if $k$ is odd and $\lambda_{1} \geq \mu_{1}$, we note that the three subcases in 1 (a) are disjoint. Certainly case (i) as well as cases (ii) and (iii) are disjoint as case (i) maps $(\lambda, \mu)$ to a 3-tuple with a primed third entry. Cases (ii) and (iii) are disjoint as $(\lambda, \mu)$ is mapped to $(\nu, a, b)$ with $a$ odd in case (ii) and $a$ even in case (iii). Similarly, we can check that the three subcases in part (b) of the bijection are distinct. So, $\phi$ is $1-1$ if $k$ is odd. Careful inspection of the definitions of $\nu$, $a, b$, and $b^{\prime}$ will show that the image of $\phi$ is the set $T_{k}$ for $k$ odd which implies $\phi$ is surjective.

While it is possible to show that $\phi$ is $1-1$ and onto, it is easier to prove $\phi$ is a bijection by constructing its inverse.

The inverse, $\phi^{-1}: T \rightarrow S$, is also defined in cases. Given $(\nu, a, b)$ or $\left(\nu, a, b^{\prime}\right)$ in $T$, first find $k$. This is easy since in all cases of the bijection $\phi$, the first part of $\nu$ is equal to $k-1$ (i.e. $\left.k=\nu_{1}+1\right)$.

1. If $k$ is odd, determine whether or not the third component, $b$, is primed.

(a) If $b$ is primed, there are three subcases:

i. If $a>b^{\prime}$ and $a$ is odd, then $\phi^{-1}\left(\nu, a, b^{\prime}\right)=\left(\left(a, b^{\prime}\right),(\nu)\right)$

ii. If $a>b^{\prime}$ and $a$ is even, then $\phi^{-1}\left(\nu, a, b^{\prime}\right)=\left(\left(a-1, b^{\prime}+1\right),(\nu)\right)$

iii. If $b^{\prime} \geq a$ then $\phi^{-1}\left(\nu, a, b^{\prime}\right)=\left((\nu),\left(b^{\prime}, a\right)\right)$

(b) If $b$ is not primed, there are three subcases:

i. If $a>b$ and $a$ is odd, then $\phi^{-1}(\nu, a, b)=((\nu),(a, b))$

ii. If $a>b$ and $a$ is even, then $\phi^{-1}(\nu, a, b)=((\nu),(a-1, b+1))$ 
iii. If $b \geq a$ then $\phi^{-1}(\nu, a, b)=((b, a),(\nu))$

2. If $k$ is even, determine whether or not the third component, $b$, is primed.

(a) If $b$ is primed, there are three subcases:

i. If $a \geq b^{\prime}$, then $\phi^{-1}\left(\nu, a, b^{\prime}\right)=\left(\left(a, b^{\prime}\right),(\nu)\right)$

ii. If $b^{\prime}>a$ and $b^{\prime}$ is odd, then $\phi^{-1}\left(\nu, a, b^{\prime}\right)=\left((\nu),\left(b^{\prime}, a\right)\right)$

iii. If $b^{\prime}>a$ and $b^{\prime}$ is even, then $\phi^{-1}\left(\nu, a, b^{\prime}\right)=\left((\nu),\left(b^{\prime}-1, a+1\right)\right)$

(b) If $b$ is not primed, there are three subcases:

i. If $a \geq b$, then $\phi^{-1}(\nu, a, b)=((\nu),(a, b))$

ii. If $b>a$ and $b$ is odd, then $\phi^{-1}(\nu, a, b)=((b, a),(\nu))$

iii. If $b>a$ and $b$ is even, then $\phi^{-1}(\nu, a, b)=((b-1, a+1),(\nu))$

Note that the set $S$ consists of pairs of partitions with no primed parts. In the definition of $\phi^{-1}$ when there is a primed integer in the image, we consider only the value of the integer and ignore the prime. We leave the details of verifying that $\phi$ and $\phi^{-1}$ are inverse maps to the reader. We conclude with two examples of $\phi^{-1}$.

Example 2.2. Let $n=6$. Given $(\nu, a, b)=\left((4,3), 2,4^{\prime}\right) \in T$. We first need to find $k$. We know $k=\nu_{1}+1=4+1=5$, so we are in the case $k$ odd. Now we need to determine which subcase, (a) or (b), contains $(\nu, a, b)=\left((4,3), 2,4^{\prime}\right)$. For the images of $\phi$ with primed entries, those with $b^{\prime} \geq a$ are in the first subcase of (a) and those with $b^{\prime}<a$ are in the second and third subcases of (b). Since we have $4^{\prime} \geq 2$ we must be in the first subcase of (a). Therefore, $\phi^{-1}\left((4,3), 2,4^{\prime}\right)=((4,3),(4,2))=(\lambda, \mu) \in S$.

Example 2.3. Let $n=7$. Given $(\nu, a, b)=\left((5,1), 0,4^{\prime}\right) \in T$. Clearly $k=6$. Since our $b$ value is primed, $a<b^{\prime}$, and $b^{\prime}$ is even we are in the third subcase of (a) for even $k$. So, $\phi^{-1}\left((5,1), 0,4^{\prime}\right)=((5,1),(3,1))=(\lambda, \mu) \in S$.

\section{Remarks}

While the $q$-analogue presented maintains some of the combinatorial properties of the classical formula, one might hope for a simpler formula. For this reason it may be of interest to investigate other $q$-analogues of the sum of cubes formula. In addition, as there are classical results for sums of other powers of integers, it would be natural to develop $q$-analogues of those theorems. To the best of our knowledge, there exist no combinatorial proofs of $q$-analogues for powers greater than three. 


\section{References}

[1] G.E. Andrews, The Theory of Partitions, Addison-Wesley, Reading, Massachusetts, 1976.

[2] A. Benjamin, M. E. Orrison, Two Quick Combinatorial Proofs of $\sum_{k=1}^{n} k^{3}=\left(\begin{array}{c}n+1 \\ 2\end{array}\right)^{2}$, The College Math Journal, 33 (2002) 406-408.

[3] G. Mackiw, A Combinatorial Approach to Sums of Integer Powers, Mathematics Magazine, 73 (2000) 44-46.

[4] R. B. Nelson, Proofs Without Words, MAA Washington DC, 1993. 